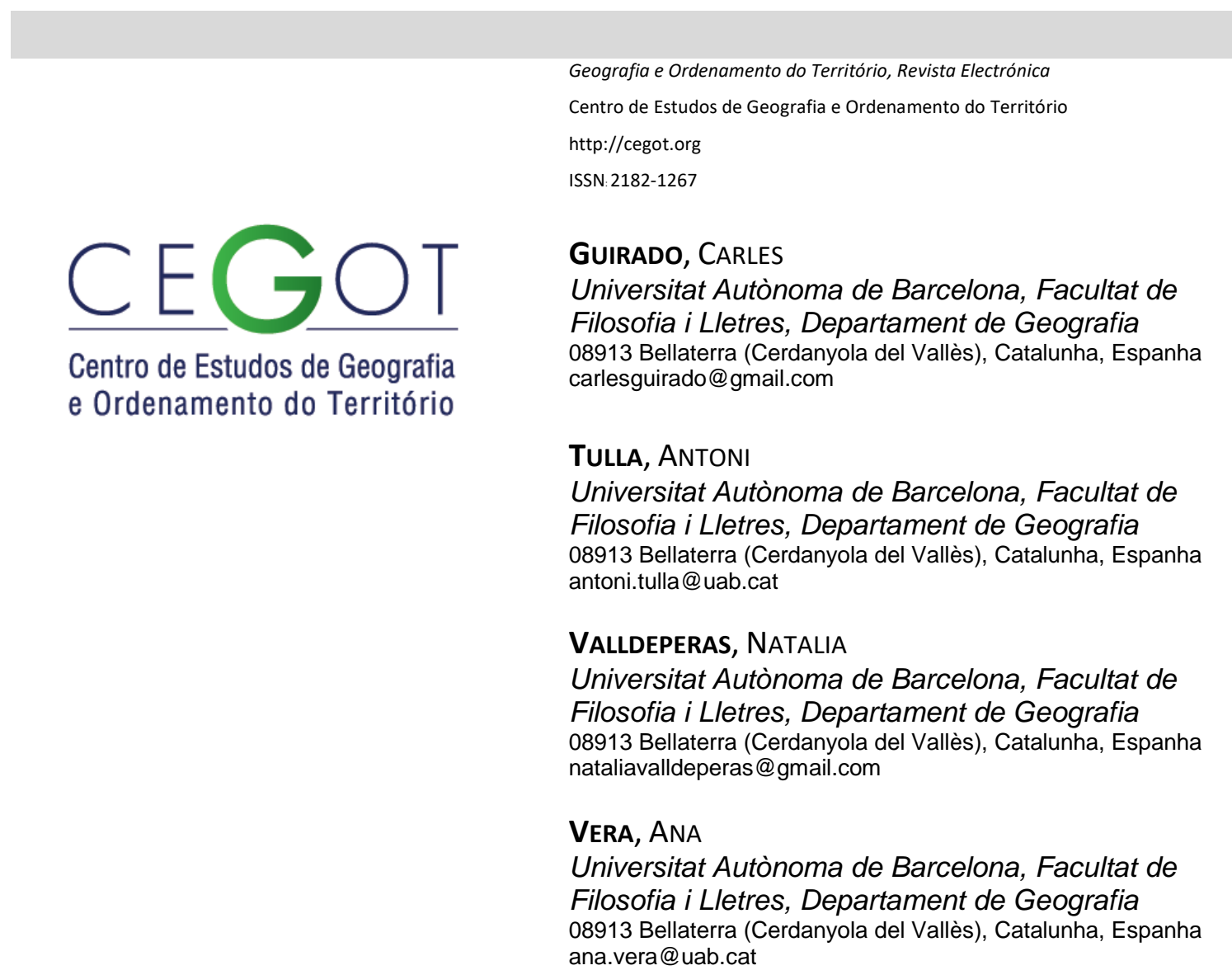

\title{
La Agricultura Social en Cataluña: una alternativa de desarrollo local sostenible frente a la crisis económica y social
}

\author{
A Agricultura Social na Catalunha: uma alternativa de desenvolvimento local \\ sustentável contra a crise económica e social \\ Social Farming in Catalonia: an alternative of local sustainable development \\ against the economic and social crisis
}

Referência: Guirado, Carles et al. (2017). La Agricultura Social en Cataluña: una alternativa de desarrollo local sostenible frente a la crisis económica y social. Revista de Geografia e Ordenamento do Território (GOT), n.o 11 (junho). Centro de Estudos de Geografia e Ordenamento do Território, p. 189-213, dx.doi.org/10.17127/got/2017.11.009

\section{RESUMEN}

La agricultura comercial busca el máximo rendimiento de la producción, lo que ha conllevado cambios muy profundos en las explotaciones agrarias y en todo el ámbito rural europeo. A causa de la denominada "Revolución Verde" han sido marginados muchos territorios que se han ido despoblando. La diversificación económica del mundo rural desde la década de 1980-89 ha impulsado la multifuncionalidad con la incorporación de las actividades turísticas y la generación de valor añadido en los productos agropecuarios. En 
paralelo ha aumentado el precio entre los consumidores por la producción agraria ecológica y de proximidad, hecho que cada vez es más correspondido por el campesinado. Desde el 2008 la crisis económica mundial ha agravado la situación de una parte importante de la población. Por un lado, cada vez se importan más productos alimentarios de baja calidad y con un coste energético imposible de mantener. Por otro, la falta de trabajo orienta a muchos jóvenes a buscar oportunidades económicas en el campo, promoviendo nuevos productos basados en criterios ecológicos y comercializados en circuitos cortos y de proximidad. En este escenario, la Agricultura Social (AS) plantea la producción y transformación de productos agrarios que implican beneficios sociales directos en términos de ocupación, formación, terapias o rehabilitación de colectivos en riesgo de exclusión social, devolviendo a la sociedad las inversiones públicas y privadas en cuatro ámbitos: a) la cohesión social; b) el empoderamiento de grupos socialmente vulnerables; c) el desarrollo local en el mundo rural y periurbano; y d) un balance más equilibrado entre ingresos y costes para la sociedad. Esta actividad emergente pretende ser un instrumento innovador en el marco de la multifuncionalidad agraria y rural.

Palabras clave: Agricultura Social, Cataluña, Desarrollo local sostenible, Exclusión social Multifuncionalidad rural

\section{RESUMO}

A agricultura comercial procurou durante décadas maximizar a produção, o que levou a mudanças profundas nas áreas rurais de toda a Europa. Por causa da chamada "Revolução Verde", muitos têm sido marginalizados e regiões inteiras fôram despovoadas. A diversificação económica nas áreas rurais desde a década de 1980-1989 impulsionou a multifuncionalidade agrária com a incorporação, por exemplo, de actividades de turismo nas explorações agrárias e geração de produtos agrícolas com valor agregado. Este fato provocou um aumento do preço os produtos agro-alimentários e a sensibilização entre os consumidores por unha agricultura orgânica, de proximidade e de preços justos. Este facto está sendo um benefício pelo campesinato, quem nalguns casos está sendo melhor retribuido pelo seu trabalho. Desde 2008 a crise económica global agravou a situação de uma grande parte da população. Por um lado, cada vez se produzem mais alimentos de baixa qualidade e com elevados custos de energia. Além disso, a falta de trabalho tem direcionado muitos jovens a buscar oportunidades económicas no campo, através da promoção de novas formas de produção e novos produtos, em base a critérios ecológicos e comercializados em circuitos locais e de proximidade. Neste cenário, a Agricultura Social (AS) aumenta a produção e transformação de produtos agrícolas que envolvem prestações sociais direitas em termos de emprego, formação, terapia ou reabilitação de grupos em risco de exclusão social, devolvendo a sociedade o investimento público e privado em quatro áreas: a) coesão social; b) empoderamento de grupos socialmente vulneráveis; c) desenvolvimento local sustentável e inovador no mundo rural e periurbano; e d) um maior equilíbrio entre investimentos e custos para a sociedade no âmbito social. Neste artigo apresentamos o caso da Catalunha através do diagnóstico do sector e análise de uma seleção de estudos de caso.

Palavras-chave: Agricultura Social, Catalunha, Desenvolvimento Local Sustentável, Exclusão social, Mulfuncionalidade agrária 


\begin{abstract}
Commercial agriculture has sought the maximum yield of production, which has led to very profound changes in farms and throughout the European countryside. Because of the socalled "Green Revolution" many territories have been marginalized and depopulated. The economic diversification of the rural world since 1980s has driven to multifunctionality with the incorporation of the tourism activities and the generation of added value in agricultural products. In parallel the price has raised among consumers for organic farming and proximity, a fact that is increasingly shared by the peasantry. Since 2008, the global economic crisis has aggravated the situation of an important part of population. On the one hand, more low-quality food with a lofty energetic cost appears in the global food system. And on the other hand, this fact and the lack of work bring many young people to seek economic opportunities in the countryside, promoting new products based on ecological criteria and marketed in local food networks. In this scenario, Social Farming (SF) proposes the production and transformation of agricultural products involving direct social benefits in terms of occupation, training, therapy or rehabilitation of groups at risk of social exclusion. Social and public investment in four areas: (a) social cohesion; (b) the empowerment of socially vulnerable groups; (c) local development in the rural and periurban world; and (d) a more well-adjusted balance between revenues and costs for society. In this paper we present the Catalonia case through the diagnosis of the sector in this territory and the analysis of a selection of study cases.
\end{abstract}

Keywords: Social Farming, Catalonia, Sustainable local development, Social exclusion, Rural Multifunctionality

\title{
1. Introducción: de la revolución verde a la multifuncionalidad
}

\section{agraria y rural}

En este trabajo pretendemos analizar como la Agricultura Social (AS) puede colaborar en el Desarrollo Local Sostenible (DLS). Esta actividad es un fenómeno emergente en los últimos 20 años, que se desarrolla en las áreas rurales y periurbanas de los países europeos y en algunos países americanos, que aporta nuevos significados a la práctica agrícola, más allá del aspecto productivo, dotándola de una función social para los colectivos en riesgo de exclusión. A parte de poder tener un empleo debe valorarse la posibilidad de que estas personas se empoderen, se sientan útiles y recuperen su dignidad. Estos colectivos hacen referencia a personas que pueden tener discapacidades físicas, mentales o psicológicas, pero también a personas drogodependientes o antiguos presos en reinserción, trabajadores en paro estructural, jóvenes inadaptados, mujeres víctimas de violencia de género jubilados, 
etc. (lacovo \& O’Connor, 2009). Asimismo la AS también es una oportunidad para la población local ya que las actividades que realiza tienen un efecto multiplicador sobre la economía al necesitar servicios asistenciales pero también por orientarse a una producción de calidad y que, muchas veces, implica una transformación de productos con el consiguiente valor añadido. La gama de actividades en la AS es muy amplia y va desde la conservación de espacios naturales o la producción agropecuaria hasta la elaboración de productos, su comercialización e incluso la restauración (Guirado et al, 2014).

El espacio rural y agrario lleva experimentando, desde hace más de medio siglo, transformaciones profundas (Woods, 2005; Woods, 2011). La reestructuración de los espacios rurales ha provocado un proceso de "mercantilización del campo" evolucionando como afirma Cloke (2006), desde los "paisajes de producción" a los "paisajes de consumo". La industrialización de la agricultura después de la Segunda Guerra Mundial, lo que algunos autores han denominado "Tercera Revolución Agrícola" (Bowler et al., 2002) o "Revolución Verde" (Lockwood, 1999) comportó importantes cambios en la función productiva de las áreas rurales, convirtiendo las explotaciones tradicionales en explotaciones competitivas adaptadas a las exigencias del mercado. Esta es una de las formas en que el sistema capitalista penetra en los territorios rurales (Arqué et al., 1982). La mecanización del campo comportó que hubiera un exceso de mano de obra, qué significó una disminución importante de la ocupación en el sector, haciendo desaparecer aquellas explotaciones "no competitivas" -principalmente las que no se podían mecanizar-con efectos irreversibles hasta hoy sobre la organiza-ción social, la cultura local y el territorio (Van der Ploeg, 2006).

Durante la segunda mitad del siglo XX la transición productivista va perdiendo impulso y se van incorporando otras funciones al territorio rural, diversificando la base económica con predominio de actividades del sector terciario (Binimelis et al., 2012). A este fenómeno se lo denomina "multifuncionalidad rural" (Armesto, 2005). La multifuncionalidad ha obligado a redefinir los programas de desarrollo rural ya que no se considera la actividad agraria como un sector económico residual, si no como una oportunidad para construir modelos económicos alternativos, los cuales no hagan depender a las sociedades rurales y su economía únicamente del turismo (Vera et al., 2011). Esta concepción de dinamización agrosocial ha hecho cambiar el papel de la agricultura y el campesinado en la sociedad actual, dando lugar al surgimiento de un nuevo agricultor con una nueva conciencia, nuevos valores y nuevas maneras de producir (Monllor, 2013), revalorizando y reconstruyendo los elementos del período más tradicional, presentes en los imaginarios y representaciones sociales sobre la ruralidad (Bell, 2006). 
Al mismo tiempo, la voluntad de tener una vida más saludable aparece como una tendencia social y de consumo emergente en las sociedades occidentales, configurándose como una alternativa al modelo alimentario impuesto por la globalización (Duch, 2010). La agricultura de proximidad, el movimiento Slow Food, o el movimiento Farm-to-table (or farm-to-fork), son conceptos similares que responden a un nuevo paradigma de alimentación, en el que, entre otros elementos, se vincula comida de calidad con el desarrollo territorial de una región. Estos planteamientos no son exclusivos de las áreas rurales, sino que recientemente también se han incluido a la agricultura urbana, relacionada con el consumo local. Los alimentos locales son los producidos por los agricultores y los ganaderos de un entorno geográfico y cultural inmediato, donde también se lleva a cabo su distribución y consumo (Halvell, 2002). El consumo de alimentos locales es una tendencia creciente que intenta evitar los efectos negativos del modelo alimenticio actual. La agricultura de proximidad pretende recuperar las actividades de las áreas rurales como la agricultura y su transformación, redistribuir los ingresos dentro de la cadena alimenticia, reduciendo los beneficios de los intermediarios y aumentando el de los agricultores locales. Se busca consumir productos frescos, más sanos y comprometer a los habitantes de las ciudades en el consumo local. Ello implica también que los recursos económicos se queden en la comunidad y se inviertan en productos de mercados locales o de la región. En este sentido, es importante tener en cuenta la teoría de la segunda mejor opción en la que toda región debe producir los bienes para la que está mejor dotada, para no ser abandonada, aunque en otra región se pueda producir a precios más bajos (Tulla et al., 2009).

La soberanía alimentaria es un derecho de las poblaciones de una región para determinar las políticas agrícolas y alimentarias que los afectan. Tales como el derecho y acceso a la tierra, a los recursos naturales, a poder alimentarse de forma sana y saludable con alimentos libres de transgénicos, a proteger y regular la producción y el comercio agrícola interior con el objetivo de conseguir un desarrollo sostenible y garantizar la seguridad alimentaria de cada región. Este concepto surgió con el apoyo del movimiento internacional "La Vía Campesina" el año 1996 cuando se realizó la Cumbre Mundial de la Alimentación de la FAO en Roma. En la actualidad, los principios de la soberanía alimentaria han sido asumidos por un amplio espectro de movimientos sociales (campesinos, mujeres, consumidores, inmigrantes, etc.). La idea principal es que los alimentos no son una mera mercancía sino un bien para el provecho de la humanidad, son un derecho (Martinez-Torres et al., 2010). La AS es una actividad que favorece la soberanía alimentaria y refuerza la agricultura de proximidad al utilizar recursos locales y orientarse a la producción ecológica. 


\section{Metodología}

La revisión bibliográfica sobre el tema de estudio y algunos temas transversales (economía social, atención social, modelos de bienestar, agricultura, desarrollo rural y local, etc.), se inició consultando las principales publicaciones científicas, diversos informes derivados de proyectos europeos, artículos académicos y publicaciones de carácter divulgativo sobre la AS en Europa y en Cataluña.

La elaboración y la corrección de la base de datos de iniciativas AS en Cataluña, en 2015 y 2016, se ha llevado a cabo a partir de un formulario web, ampliando el ámbito de actuación de las entidades y considerando no tan solo la inserción sociolaboral sino también la terapia, la rehabilitación, la educación y la formación. Esto ha permitido elaborar un mapa colaborativo que pretende, aparte de mostrar la implantación de la AS en Cataluña, reforzar el nexo y la red dentro del sector y al mismo tiempo intentar involucrar tanto a los actores principales del sector como a la ciudadanía en general. Actualmente, hay 150 entidades en la base de datos, sabiendo que 105 desarrollan AS, de las cuales 78 realizan exclusivamente actividades de AS y 27 de ellas lo combinan con la jardinería. De las 45 entidades de jardinería, que las tenemos en cuenta por su potencialidad en la AS, 31 realizan propiamente actividades de jardinería y 14 lo combinan con otras actividades.

El análisis cualitativo que se ha realizado de la AS ha sido a partir de las 19 entrevistas semiestructuradas en profundidad a las iniciativas AS, los 3 cuestionarios vía telemática a promotores y/o responsables de iniciativas de AS, que hemos denominado informantes clave, y el trabajo de campo. Para el análisis fue necesaria la transcripción literal de las entrevistas y el vaciado de los cuestionarios que se combinaron con la información obtenida del trabajo de campo. El resultado que se obtuvo fue una matriz de datos que definía de forma sintética y ordenada las principales características de las iniciativas de AS en Cataluña. El análisis de las entrevistas permitió elaborar un detallado análisis de las debilidades, amenazas, fortalezas y oportunidades (DAFO) de los factores que intervienen en el funcionamiento del sector y de las entidades de AS. A partir de estos resultados se elaboró un flujograma o mapa de elementos que permitió focalizar cuales son los elementos clave en el desarrollo de la actividad. Poder identificar estos factores sirvió para elaborar una serie de recomendaciones estratégicas para el impulso de AS en Cataluña (Tulla et al, 2015). 
Finalmente, y con el objetivo de establecer las principales características de las entidades estudiadas y profundizar en el análisis del sector de la Agricultura Social en Cataluña se ha utilizado la metodología CANVAS o modelo de negocio. El Business Model CANVAS es una herramienta metodológica que permite analizar y visualizar de forma clara, ágil y sencilla la propuesta y la cadena de valor de cualquier iniciativa, ayudando a definir un modelo de negocio rentable y sostenible. Esta metodología se ha aplicado a diez casos de estudios que han sido seleccionados por la tipología del espacio en el que se ubican. Estos criterios se explican con mayor detalle en el apartado 5.

\section{La Agricultura Social: una alternativa para el desarrollo local sostenible}

La AS podríamos definirla como un proceso de integración y empoderamiento de colectivos en riesgo de exclusión social, a través de participar en las actividades agrarias y su transformación. La integración incluye la creación de lugares de trabajo o de formación de las personas de los citados colectivos, junto a medidas terapéuticas si son necesarias. Las entidades, su forma jurídica, el financiamiento y la colaboración entre las instituciones públicas y privadas dependen del sistema de salud y de las políticas sociales de cada país (Tulla et al., 2014). En el norte de Europa se utiliza el término Green Care (GC) que teniendo el mismo objetivo de empoderamiento utilizando las actividades agrarias, se diferencia de la AS por priorizar el uso de terapias curativas, el medio natural como paliativo y una relación clientelar entre las entidades gestoras y las personas de los colectivos en riesgo de exclusión social, básicamente en dificultades especiales o diversidad funcional (Leck et al., 2014). Las actividades de la AS también benefician a la población local, al facilitar la creación de servicios sociales y de salud, así como generar multifuncionalidad y valor añadido con actividades de transformación y comercialización de productos agroalimentarios (Guirado et al., 2014).

En el presente contexto de crisis socioeconómica, la AS aparece como una clara muestra de innovación social, estrechamente relacionada con la acción colectiva, la cohesión y la interacción activa de la personas con los recursos locales como mecanismo para una inserción laboral real, independientemente de su condición social. La agricultura, y por extensión el resto de subsectores agrarios, precisa de un vínculo con el territorio que 
contribuye a hacer de esta práctica un elemento clave en el desarrollo local de territorios rurales y periurbanos, vertebrando la gestación de proyectos innovadores, creando nuevas oportunidades de ocupación -tanto para personas en riesgo de exclusión como para la población en general-, tejiendo redes de cooperación y ayudando a fijar población al territorio y ampliando la dotación de servicios en estas áreas, en las que a menudo hay déficit de centros y profesionales sociosantarios que atiendan las necesidades de los colectivos más vulnerables.

Esta nueva agricultura apuesta por un modelo agroalimentario más integrado socialmente que vela por el acceso directo a productos ecológicos, con la mínima carga energética posible y con valor social añadido. Los consumidores acceden a este nuevo modelo a través de la compra directa en la explotación, grupos y cooperativas de consumo y establecimientos especializados, materializando un consumo responsable como acción consciente de crítica y denuncia del sistema agroalimentario imperante y reivindicando la soberanía alimentaria de los pueblos (López y Fernández, 2003; Carbone et al., 2007; Schneider, 2008). El cambio en la situación de los territorios rurales, junto con la emergencia de un nuevo campesinado, las innovaciones sociales y las nuevas tendencias en el sector de los servicios, han contribuido a tejer nuevas alianzas entre agricultura y sociedad. En este contexto, la AS emerge como una estrategia para ofrecer soluciones y respuestas a problemas y necesidades presentes en la sociedad actual, las cuales surgen en la mayoría de las ocasiones desde la implicación y participación de la ciudadanía, con el fin de subsanar las carencias (y a menudo también la ineficiencia) de las políticas públicas en la esfera de lo social, del desarrollo rural y local y de las políticas agrarias.

\section{La Agricultura Social en Cataluña: diagnosis del sector}

El desarrollo de la AS en Cataluña se puede desglosar en cuatro etapas. La primera se sitúa entre la década de 1960 y la de 1970, época en la que surgen las primeras iniciativas de AS en el área circundante de Barcelona, ubicándose en dos lugares clave de la agricultura periurbana: el Delta del Llobregat y el Maresme. Entre mediados de la década de 1970 e inicio de la década de 1990, empiezan a gestarse algunos proyectos en lugares más alejados del Área Metropolitana de Barcelona y aparecen iniciativas en territorios rurales periféricos, con mayor presencia del sector agrario, como por ejemplo en las comarcas de la Segarra, el 
Segrià, el Solsonès y la Garrotxa. Esta segunda etapa coincide con una profunda crisis del sector agrario y con el auge del movimiento neorural, hecho que cabe destacar ya que algunas de las iniciativas surgidas durante esta etapa fueron desarrolladas por personas de origen urbano que decidieron migrar al campo en busca de un nuevo estilo de vida y la posibilidad de desarrollar proyectos comunitarios (Nogué, 1982; Guirado, 2011). Se trata de áreas con escasa población, una estructura sociodemográfica mellada por la despoblación iniciada a mediados del siglo XIX y una economía fuertemente polarizada entre un sector agrario frágil y una importante dependencia del sector turístico (Barrachina et al., 2009; Guirado y Tulla, 2010), donde los proyectos de emprendimiento social tienen un encaje difícil (Pallarès-Blanch et al., 2015). También podemos destacar que son áreas con un importante déficit en la dotación de servicios, especialmente en el ámbito sociosanitario, lo que provoca una migración hacía la ciudad de estos sectores de población más vulnerables, donde pueden recibir una atención de mayor calidad y especializada.

El inicio de la tercera etapa la hemos situado en 1992, con un desempleo alrededor del $24 \%$, a raíz de la crisis económica acaecida en España. En este contexto surgieron iniciativas de AS con la finalidad de atender a los colectivos más vulnerables y para reducir las desigualdades sociales inducidas por la situación socioeconómica del momento. La recuperación de la situación económica a finales de la década de 1990 fue el marco propicio para el desarrollo de iniciativas de carácter social, ya que permitió a las administraciones públicas destinar parte de los presupuesto a finalidades sociales, aumentando la dotación económica y el número de subvenciones y ayudas al Tercer Sector Social (TSS). Estas iniciativas se localizan próximas a las principales capitales catalanas pero se expanden hacía zonas periféricas.

La cuarta etapa, empieza en 2008, con la actual crisis económica y financiera. En este contexto aumenta la creación de proyectos de AS, para dar respuesta a la pérdida de capacidad de reacción de los gobiernos frente a las necesidades sociales, a la generación de redes de ayuda ciudadana y a la construcción de alternativas económicas. Algunos proyectos se sitúan en zonas rurales alejadas de los centros urbanos, evidenciando que esta práctica es percibida por algunos emprendedores/as rurales como una estrategia de desarrollo válida para estos territorios, a la vez que hacen frente a la crisis con proyectos innovadores, con valor social y en los que el uso de recursos locales es primordial. Algunas fundaciones privadas establecen redes de ayuda y colaboración entre los proyectos de AS.

Actualmente hemos detectado la existencia de más de un centenar de experiencias que se dedican principalmente a la creación de oportunidades de ocupación de colectivos en riesgo de exclusión social (70\%), aunque también encontramos otras que desarrollan su actividad 
en el ámbito de la terapia o la rehabilitación (3\%), la educación y la formación (5\%) o a la gestión de huertos sociales (23\%). La mayor parte de estas experiencias se dedican propiamente a la agricultura (74\%), aunque también encontramos actividades de transformación agroalimentaria (10\%), servicios (7\%) vinculados a la actividad agraria, como pueden ser la comercialización a través de circuitos cortos, a la artesanía (1\%) y a la ganadería (2\%). En cuanto a las modalidades de inserción de los centros, la más extendida son los Centros Especiales de Empleo (CEE) que representan un 43\%, seguidos de las Empresas de Inserción (EI) (17\%), de las Asociaciones (11\%), los Centros Ocupacionales (2\%). Cabe destacar que hay un grueso de entidades sin una modalidad de inserción específica (27\%), por falta de normativa o legislación, y que mayoritariamente hace referencia a los huertos sociales.

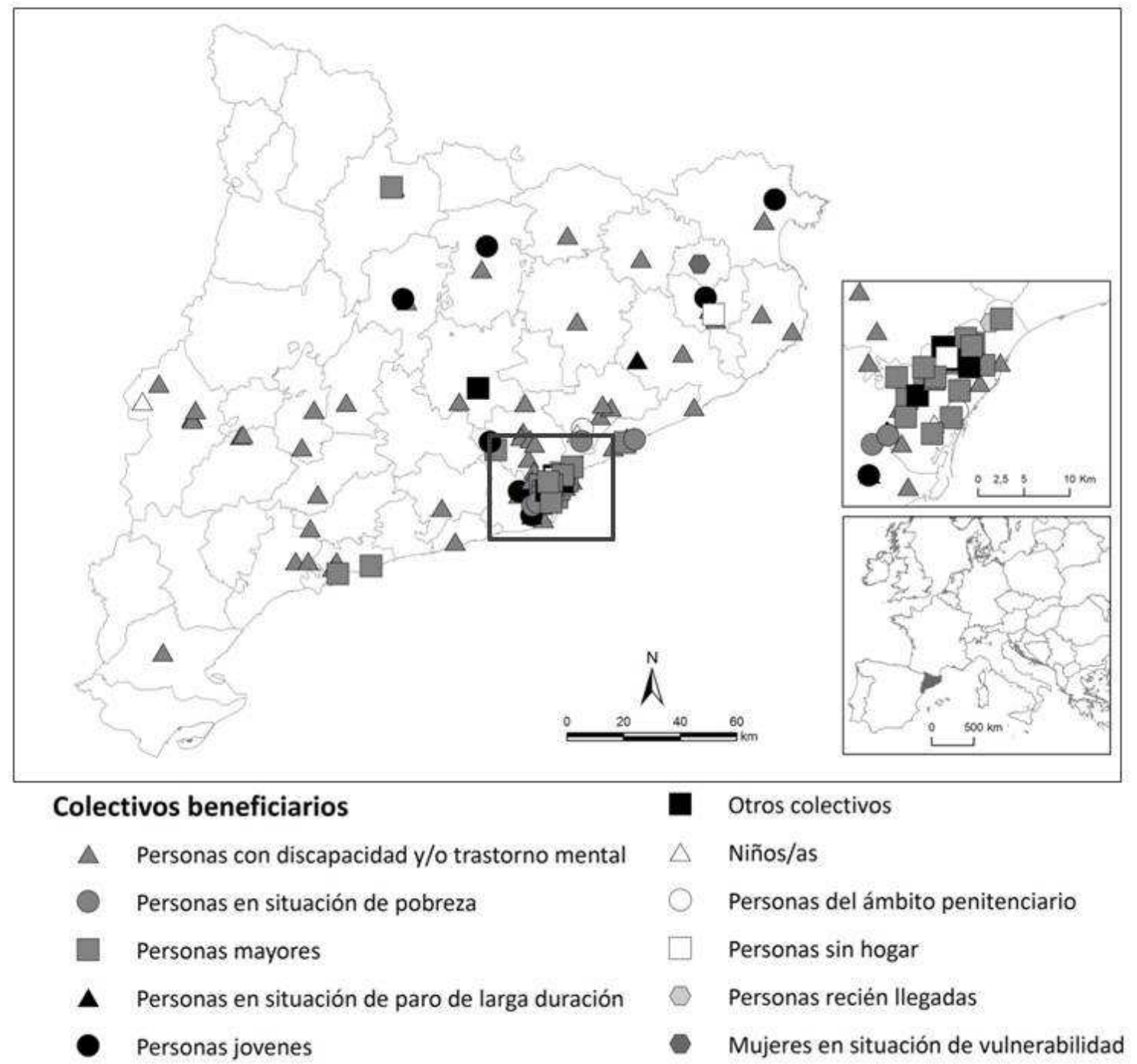

Figura 1 - Distribución territorial de la Agricultura Social en Cataluña (2015). (Fuente: Elaboración propia sobre la Base de Datos creada por el equipo ERAS-UAB) 
Si nos fijamos en el volumen de trabajadores/as de los colectivos de personas beneficiarias observamos como la mayor parte de las iniciativas de AS tienen plantillas en las que al menos un 50\% están formadas por personas en riesgo de exclusión social, llegando a cifras en torno al $80 \%$ y $90 \%$ sobre el total de trabajadores en algunos casos. Esto es un fenómeno que se explica por la aplicación de la legislación vigente, ya que según la ley las plantillas de los Centros Especiales de Empleo (CET) han de estar formadas, como mínimo, por un 70\% de personas con discapacidad. En el caso de las Empresas de Inserción (EIns), oscila entre 30\% y $70 \%$, lo que conlleva que en esta modalidad de inserción el porcentaje de personas beneficiarias sea ligeramente inferior que en el caso de los CET (Figura 1).

En este proceso se han clasificado los distintos factores que intervienen en el funcionamiento del sector y de las entidades de AS, en debilidades, amenazas, fortalezas y oportunidades. Poder identificar estos factores nos ha permitido elaborar las etapas posteriores de análisis, y muy especialmente el diseño de las recomendaciones para una potenciación de esta actividad en Cataluña (Tulla et al., 2015). Se detectaron, por un lado, los problemas más importantes que dificultaban el desarrollo de la AS, y por el otro, los elementos favorables que lo beneficiaban, tal como se expone en la tabla 1. Las fortalezas se relacionan con el desarrollo de la actividad agraria en el propio territorio, combinando con la innovación social y empresarial, dando servicio a colectivos en riesgo de exclusión social, siempre y cuando las instituciones tengan un papel de facilitador activo y de promotor y la sociedad civil encabece la creación de nuevas experiencias.

En cambio, las debilidades las encontramos en la frágil organización de las experiencias de AS y en el poco conocimiento que tiene la sociedad de las mismas. En el actual contexto de crisis económica y financiera, se han reducido las ayudas institucionales y la ausencia de una legislación clara es una amenaza para el desarrollo futuro de la AS. En cualquier caso, el problema principal es la falta de voluntad política para promocionar estos proyectos. Situación que se ha suplido con la creciente participación del Tercer Sector Social (TSS), tanto en la organización (cooperativas, sociedad limitada laboral, etc.) como en la promoción (fundaciones, asociaciones, etc.). 


\begin{tabular}{|c|c|}
\hline FORTALEZAS & OPORTUNIDADES \\
\hline $\begin{array}{l}\text { AGRICULTURA } \\
\text { - } \quad \text { Agricultura, sector estratégico ante la crisis } \\
\text { - } \quad \text { de las explotaciones agrarias } \\
\text { - } \quad \text { Proximidad alimentaria y dignificación práctica agrícola } \\
\text { - } \quad \text { Implementación de criterios agroecológicos y valor social } \\
\text { de las explotaciones } \\
\text { TERRITORIO } \\
\text { - Contribución al desarrollo local y a la cohesión social } \\
\text { - } \quad \text { Creación de servicios de atención a las personas } \\
\text { - } \quad \text { Nuevas relaciones entre productores, consumidores y la } \\
\text { INNOVACIÓN SOCIAL Y EMPRENDEDURÍA } \\
\text { - } \quad \text { Retorno de la inversión pública a la sociedad } \\
\text { - Iniciativas de origen cívico } \\
\text { - } \quad \text { Fomento de la cooperación público-privada } \\
\text { BENFICIARIOS } \\
\text { - } \quad \text { Adecuación del empleo a colectivos específicos } \\
\text { - } \quad \text { Apoyo a persones en riesgo de exclusión social } \\
\text { - Beneficios para los colectivos de usuarios }\end{array}$ & $\begin{array}{l}\text { CONTEXTO ACTUAL } \\
\text { - } \quad \text { Escenario socioeconómico que obliga a la } \\
\text { innovación y proyectos con valor social } \\
\text { - } \quad \text { Cambios en las pautas de consumo (productos } \\
\text { justos y éticos) y de los hábitos alimentarios } \\
\text { (productos ecológicos) } \\
\text { EMPLEO E INCLUSIÓN SOCIAL } \\
\text { - } \quad \text { Nuevos yacimientos de empleo en la agricultura y } \\
\text { - los sectores derivados } \\
\text { Necesidad de crear iniciativas que generen empleo } \\
\text { SOCIEDAD } \\
\text { - } \quad \text { Fuentes de financiación alternativa } \\
\text { - Uso de nuevas tecnologías } \\
\text { - } \quad \text { Amplia red del Tercer Sector Social en Cataluña } \\
\text { INSTITUCIONAL } \\
\text { - } \quad \text { Programas de fomento de la economía social } \\
\text { - } \quad \text { Ayudas al emprendimiento y a la innovación }\end{array}$ \\
\hline DEBILIDADES & AMENAZAS \\
\hline $\begin{array}{l}\text { GESTIÓN/ORGANITZACIÓN } \\
\text { - } \quad \text { Dependencia del financiamiento público } \\
\text { - } \quad \text { Bajos rendimientos productivos } \\
\text { ENTORNO } \\
\text { - } \quad \text { Sector emergente con poca trayectoria } \\
\text { - Práctica poco codificada } \\
\text { - } \quad \text { Redes poco estructuradas } \\
\text { BENFICIARIOS/AS } \\
\text { - Baja presencia de determinados colectivos } \\
\text { - Dificultades para solucionar las necesidades específicas } \\
\quad \text { de colectivos/individuos en riesgo de exclusión social }\end{array}$ & $\begin{array}{l}\text { CONTEXTO } \\
\text { - } \quad \text { Incerteza y reticencias para desarrollar el } \\
\text { - } \quad \text { Falta de conciencia y reconocimiento del trabajo } \\
\text { social } \\
\text { - } \quad \text { Modelo neoliberal (objetivos de empresa) vs } \\
\text { economía ética y solidaria. } \\
\text { SITUACIÓN AGRICULTURA SOCIAL } \\
\text { - } \quad \text { Escasa valoración de la aportación social de los } \\
\text { - } \quad \text { Escasoctivos de AS y en riesgo de exclusión social } \\
\text { POLÍTICA/INSTITUCIONto de la AS en Cataluña, en } \\
\text { - } \quad \text { Recortes en materia de bienestar social } \\
\text { - Socialización y privatización de las competencias de } \\
\text { - } \quad \text { atención sociosanitaria } \\
\text { - } \quad \text { Falta de voluntad política } \\
\text { - Marco normativo inexistente }\end{array}$ \\
\hline
\end{tabular}

Tabla 1 - Características del DAFO de la Agricultura Social en Cataluña. (Fuente: elaboración propia) 
A partir de la última versión del análisis DAFO se elaboraron dos flujogramas, en los que se colocaron, por una banda, todos los elementos o factores "favorables" (oportunidades y fortalezas) para el desarrollo de la AS en Cataluña $y$, por otra, todos los elementos "limitantes" (debilidades y amenazas). Estos elementos se colocaron en dos ejes: el vertical, que correspondía a la escala de actuación o de influencia de cada factor (proyecto, sector o ámbito externo), y el horizontal, que se refería a grandes ámbitos de actuación como la información y la comunicación, los recursos y las infraestructuras, la gestión y organización y la sociedad. De esta manera, los elementos o factores se podrían disponer en un lugar en el flujograma en función de la escala en la que operaban y del ámbito temático al que hacían referencia, dotándolos de características que posteriormente serian de utilidad para abordar posibles acciones estratégicas.

El resultado de ubicación de los elementos fue un diagrama muy denso, que tuvimos que sintetizar agrupando los factores en categorías para facilitar la lectura de los mapas y la posterior creación de conexiones entre ellos (Figura 2). Esta agrupación se realizó por proximidad temática.

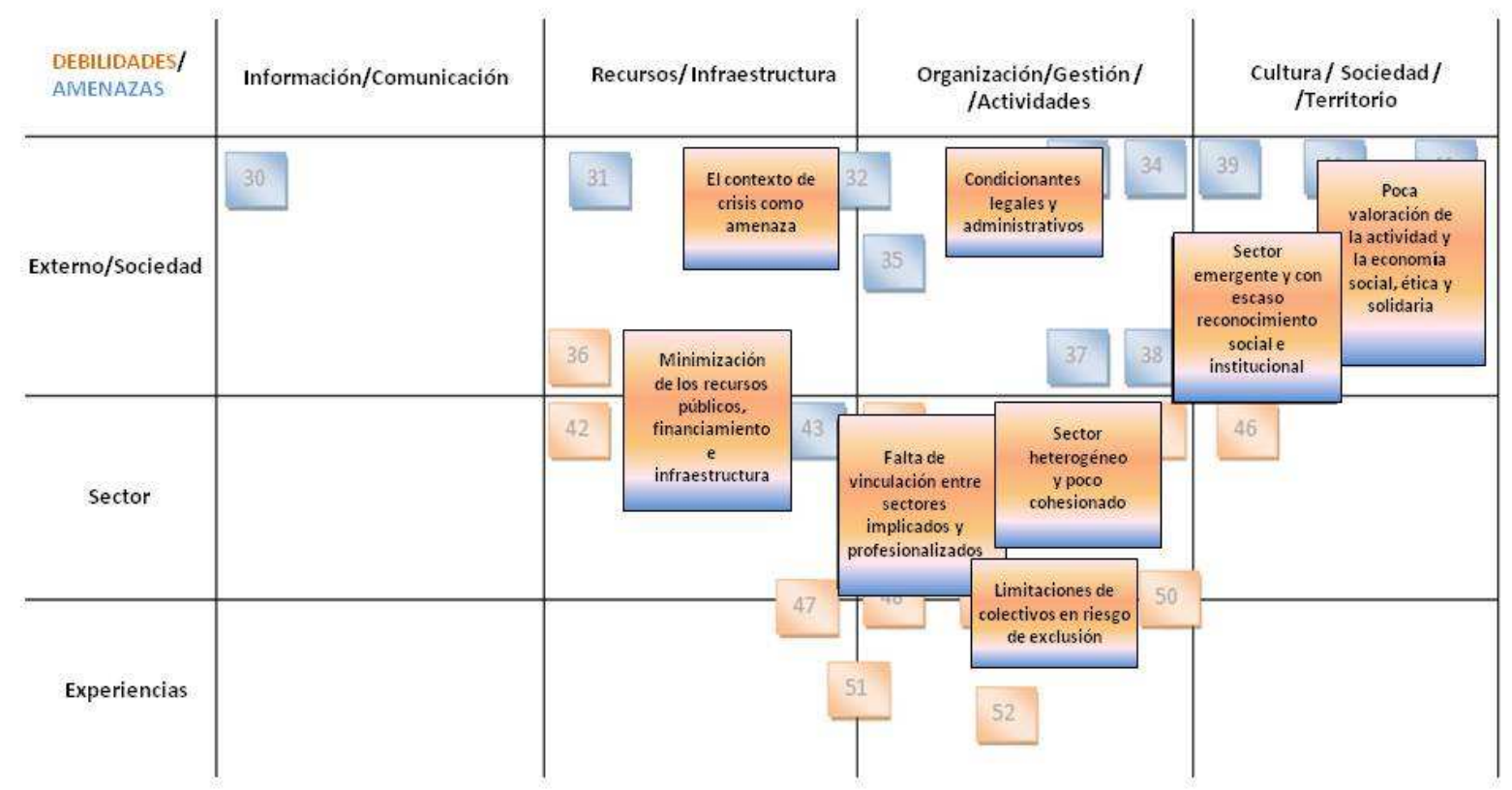

Figura 2 - Flujograma de Debilidades y Amenazas (Fuente: Elaboración propia)

Una vez realizado el proceso de síntesis de los flujogramas procedimos a establecer conexiones entre las diferentes categorías. Estos vínculos pueden ser unidireccionales, haciendo referencia a relaciones causa-efecto, o bidireccionales, en el caso que los elementos sean mutuamente influenciables. El resultado es una red de elementos interconectados entre ellos por relaciones de causalidad e interdependencia, formando así 
un sistema que nos proporciona una visión integral y completa del sector de la AS, en el cuál están representados des de los factores que condicionan cada uno de los proyectos de AS hasta los elementos externos que influyen en el contexto y que determinen.

De las relaciones resultantes se contabilizan las entradas (flechas que llegan) y las salidas (flechas que salen) de cada uno de los elementos, lo que nos permite saber la importancia y la posición que ocupa cada uno de los nodos en este sistema. Este método, basado en el análisis de redes, permite jerarquizar y priorizar los elementos presentes en los flujogramas. De este modo, los nodos que tienen más salidas son los elementos más determinantes, que tienen mayor poder para condicionar el devenir del sector en un futuro a corto-mediano plazo, y los que tienen más entradas los más condicionados por el resto de elementos, que acostumbran a ser consecuencias o efectos de los elementos más determinantes. Esto nos ha permitido establecer en ambos flujogramas diferentes niveles de análisis que van desde los elementos que se configuran como contexto hasta aquellos que caracterizan el sector y las experiencias y que determinan la dinámica del fenómeno en Cataluña (Tabla 2).

\begin{tabular}{|c|c|}
\hline FLUJOGRAMA 1: FORTALEZAS/OPORTUNIDADES & FLUJOGRAMA 2: DEBILIDADES/AMENAZAS \\
\hline $\begin{array}{l}\text { Contexto: Elementos favorables que hacen } \\
\text { referencia al contexto socioeconómico y a las } \\
\text { pautas sociales y culturales predominantes }\end{array}$ & $\begin{array}{l}\text { Contexto: Elementos desfavorables que hacen } \\
\text { referencia al contexto socioeconómico y a las } \\
\text { pautas sociales y culturales predominantes }\end{array}$ \\
\hline $\begin{array}{l}\text { Nivel 1: Factores externos que contribuyen a crear } \\
\text { un entorno propicio por el desarrollo del AS en } \\
\text { Cataluña }\end{array}$ & $\begin{array}{l}\text { Nivel 1: Problemas clave y factores externos que } \\
\text { limitan el desarrollo de la AS }\end{array}$ \\
\hline $\begin{array}{l}\text { Nivel 2: Elementos propios de los sector que } \\
\text { muestran las oportunidades del AS como práctica } \\
\text { innovadora en el ámbito social y agrario }\end{array}$ & $\begin{array}{l}\text { Nivel 2. Carencias y limitaciones del sector para } \\
\text { desarrollar correctamente al suya actividad }\end{array}$ \\
\hline $\begin{array}{l}\text { Nivel 3. Efectos que tendría la expansión y el } \\
\text { fomento del AS en la cohesión social y en la } \\
\text { situación de los colectivos en riesgo de exclusión }\end{array}$ & $\begin{array}{l}\text { Nivel 3. Consecuencias y efectos que tienen los } \\
\text { factores desfavorables y problemas clave sobre el } \\
\text { sector de la AS y las entidades. }\end{array}$ \\
\hline
\end{tabular}

Tabla 2 - Categorías resultantes del análisis de redes de elementos. (Fuente: Elaboración propia)

\section{Casos de estudio}

En áreas donde la emigración campo-ciudad ha sido muy fuerte, la AS ha colaborado a un cierto equilibrio territorial evitando la exclusión social de estos territorios y ayudando a favorecer la creación de servicios de atención social y sanitaria en áreas rurales periféricas. 
Además de esto es un catalizador de alternativas económicas, fundamentadas en una nueva concepción de la agricultura, ecológica y de proximidad, pero también de la colaboración con otras actividades de un área determinada. En las áreas periurbanas, estos beneficios han permitido recuperar terrenos abandonados, hacer retroceder las áreas forestales y dar una orientación económica viable a muchos proyectos de AS. En nuestra investigación hemos seleccionado diez proyectos en Cataluña que abarcan 3 casos en el periurbano de Barcelona (PEB), 3 en municipios rurales (MUR) y 4 en el ámbito rural con fuerte dependencia urbana (RUB).



Figura 3 - Mapa de la ubicación de las entidades seleccionadas (Fuente: elaboración propia)

L'Ortiga (PEB) es una cooperativa con dos líneas, una encargada de la producción y comercialización agraria ecológica de una finca de 2 ha, básicamente hortalizas, y otra, de iniciativa social sin ánimo de lucro, en la que se desarrollan actividades de educación y formación, en colaboración con los Servicios Sociales del Ayuntamiento de Sant Cugat del Vallès para la inserción de colectivos en situación de pobreza. También realizan actividades vinculadas a la agricultura para escuelas y formación para adultos, y subministran cestas ecológicas a grupos de consumo responsable y a clientes particulares a través de internet. 
Esta iniciativa surge el año 2010 en la finca de Can Montmany (Valldoreix), situada en la Parque Natural de Collserola y cedida por el ayuntamiento.

Masia Can Calopa de Dalt (PEB) es una cooperativa encargada de trabajar y gestionar las 3 ha de viñedos de una finca propiedad del Ayuntamiento de Barcelona situada en el Parque Natural de Collserola, y se crea en 2010 relacionada con el proyecto L'Olivera Cooperativa, una de las iniciativas de AS más consolidadas, creada en 1974 en Vallbona de les Monges (Lleida). Su objetivo es cualificar a jóvenes del mundo urbano, sin formación previa, mediante la actividad laboral agrícola, junto con el apoyo formativo que reciben y la vida en un hogar social en un entorno agrícola y natural. Ocupa a 11 jóvenes que desarrollan trabajos agrícolas en otras fincas dentro del Área Metropolitana de Barcelona, además de las de la masia.

Els Horts de Can Salas (PEB) son un proyecto del Centro de Jardinería l'Heura, SLL, de Terrassa, fundado en 1994 como entidad no lucrativa de inserción sociolaboral para personas con discapacidad psíquica y/o trastorno mental como centro de jardinería y vivero de plantas. Desde 2013 realizan una producción ecológica de verduras y hortalizas en la finca de Can Salas (Terrassa), cultivando 1 ha, que ahora ampliarán a 3 por el éxito de ventas. Disponen de una agrotienda desde la que también suministra "cestas" a grupos de consumo, realizan venta directa y ofrecen catering. Han potenciado los canales cortos de distribución y han desarrollado un programa educativo para escuelas y talleres de formación para familias.

Aprodisca Ambientals i Ecologics (MUR) es una El, promovida en 2008 por la Asociación Pro Personas con Disminución Psíquica de la Conca de Barberà (APRODISCA), como proyecto social que se desarrolla en el sector agroalimentario ecológico. Está orientada al cultivo de verduras y hortalizas, ocupando a personas en riesgo de exclusión social. Con los productos de la huerta se realizan otras actividades complementarias impulsadas desde APRODISCA, como el proyecto Hortus Aprodiscae, en el cuál las personas con discapacidad intelectual del CET APRODISCA preparan lotes de productos ecológicos, y otros productos elaborados (salsas y condimentos). También realiza cátering para eventos.

Fundació Casa Dalmases (MUR), creada en 2009 para gestionar el espacio de la casa señorial que lleva el mismo nombre, con el doble objetivo de dar oportunidades a personas con discapacidad intelectual mediante la producción de cerveza artesanal, y abrir al público el patrimonio de una parte de la casa, donde también se desarrollan actividades sociales y culturales. La planta baja de Casa Dalmases tiene una tienda que emplea a personas con discapacidad de un centro ocupacional y vende productos de otras entidades sociales de la 
comarca. Participa en mercados del entorno ocupando personas excluidas socialmente, como personas sin hogar. En el 2015 inició una nueva línea de negocio centrada en la producción de chocolate artesanal, un proyecto en colaboración con entidades sociales y productores de Ecuador.

Sambucus (MUR) es una cooperativa creada el año 2010 en Manlleu (Barcelona), para proporcionar los recursos necesarios de empoderamiento a las personas en riesgo de exclusión social, colaborando con los servicios locales de los municipios del entorno. Realizan producción de huerta ecológica, gestionan un restaurante en el Mercado Municipal, cultivan plantas aromáticas en ecológico, que ellos mismos secan en sus instalaciones y comercializan en tiendas especializadas. También cocinan para comedores de instituciones (escuelas, hospitales, etc.) y ofrecen servicios de catering. Este proyecto integral se basa en la idea de conectar y controlar todo el ciclo productivo, desde el origen del producto hasta el consumidor final.

RiuVerd (MUR) es una cooperativa de iniciativa social sin ánimo de lucro creada en 2011. Su objetivo es integrar y formar social y laboralmente a personas en situación de exclusión social como tránsito al empleo ordinario, en especial jóvenes, a quienes se ofrece una formación personalizada adaptada a sus necesidades y circunstancias personales. Desarrollan otras actividades económicas como la implementación de un comedor colectivo saludable, el cultivo de productos hortícolas ecológicos y de calidad, su distribución y venta en cestas, el cultivo de hierbas aromáticas, servicios de jardinería y otros servicios del hogar.

Bolet Ben Fet (RUB), creado en el 2009, es la colaboración entre un emprendedor y el Grupo cooperativo TEB, una organización centrada en la búsqueda de oportunidades para las personas con discapacidad intelectual. El objetivo es la inserción social y laboral de personas con discapacidad intelectual a través de un CET destinado al cultivo de setas de producción ecológica del tipo shitake y maitake. Esta actividad se lleva a cabo en una antigua granja en Sant Antoni de Vilamajor (Vallès Oriental).

La Klosca (RUB) surgió el 2009 para la producción de huevos ecológicos en la finca de Sant Miquel de Mata, cedida por el Ayuntamiento de Mataró. Iniciativa creada a través de la entidad social Centre de Prevenció $i$ Treball, que tiene por objetivo completar el círculo asistencial (asistencia social, sanitaria y ocupación) de las personas afectadas por trastorno mental severo con las que trabaja. Se realiza una producción de calidad con la función social de conseguir la inserción sociolaboral de las personas beneficiarias. Esta entidad es una reconversión a AS de viveros de plantas aromáticas y ornamentales. 
Delícies del Berguedà (RUB), marca comercial de la empresa de inserción Portal Berguedà promovida por la Fundación Portal, se constituyó el 2011. Esta organización ayuda a jóvenes con patología dual y a sus familias en la comarca del Berguedà. Su actividad principal es la producción de derivados lácteos como yogures y queso, mediante materia prima local, procedente de una granja del entorno, favoreciendo así el consumo local, las sinergias entre iniciativas de un mismo territorio y el desarrollo rural sostenible.

Hemos podido comprobar que uno de los elementos que más nos ayuda a clasificar las entidades seleccionadas es la forma jurídica que utilizan. De las 10 entidades, 5 son sociedades mercantiles sin ánimo de lucro (Sociedad Limitada, Sociedad Limitada Unipersonal o Sociedad Limitada Laboral), 4 son cooperativas y una es una fundación. Esto indica que, aunque todas ellas tengan una función social y asistencial, las iniciativas tienen orientación mercantil, y que la prioridad es realizar una actividad económica que sea viable para poder llevar a cabo sus principales objetivos en la esfera social. Otra característica es la consideración como CET o El, aprovechando las ventajas de la legislación sobre entidades que dan empleo a personas en riesgo de exclusión social. Es importante destacar que 8 de las 10 entidades realizan agricultura siguiendo criterios ecológicos con sello de la autoridad certificadora en Cataluña, el Consell Català de la Producció Agrària Ecològica (CCPAE) o están en conversión para obtenerlo. Una de las entidades produce ecológicamente sin sello de una entidad certificadora y otra no puede realizar la totalidad de su producción con criterios de agricultura ecológica porque las condiciones del cultivo y las exigencias del terreno no lo permiten. Podemos concluir entonces que la voluntad de todas las entidades de AS analizadas es la de producir ecológicamente aunque en algún caso particular todavía no es posible, situación que es común con el resto de entidades del sector.

\section{Resultados}

La AS puede considerarse una actividad que quiere mejorar las características de la agricultura de mercado, buscando nuevas oportunidades en el tipo de producción, en la creación de valor añadido, en las formas de comercialización y, muy especialmente, en las sinergias con otras actividades del territorio. Se debe considerar que el hecho de trabajar con colectivos en riesgo de exclusión social no quiere decir que la AS sea una actividad marginal. Precisamente, se está demostrando que los proyectos de AS son viables económicamente y consiguen un retorno social, económico y ambiental, superior al de los 
recursos utilizados. Uno de los elementos determinantes es la colaboración entre las entidades de AS, en formación, ayuda mutua y muy especialmente en la comercialización.

Por lo que se refiere a la estrategia en el mercado se identifican dos tipos de ventaja competitiva (Porter, 1985). Una, la basada en el coste, cuando una empresa es capaz de ofrecer un producto o servicio con los mismos beneficios que la competencia pero a un precio más bajo, y dos, la basada en la diferenciación, cuando una empresa tiene la capacidad de ofrecer un producto o servicio, los beneficios del cual superan los de la competencia. En general todas las entidades de AS compiten en el mercado por diferenciación, ofreciendo productos y servicios de calidad superior a los de sus competidores directos o indirectos. Sin embargo, el $50 \%$ de estas compiten en alguna de sus líneas de negocio o para algún segmento de mercado por coste, ofreciendo precios competitivos a sus clientes para que estos decidan comprar sus productos.

En segundo lugar, el argumento principal de venta para todas las entidades es la calidad de los productos, valor que está en todos los casos por delante del proyecto social. En las entrevistas, los promotores de las iniciativas seleccionadas han comentado que el proyecto social que hay detrás de los productos nunca se vende como un valor de compra, ya que esto no garantiza una estabilidad y un lugar competitivo en el mercado. El proyecto social, en todos los casos, aseguran las personas entrevistadas, debe ser un refuerzo de compra; una opción más, preferiblemente secundaria, por la que las personas consumidoras decidan apostar por el producto. Estas entidades también apuestan por una relación personalizada y directa con sus clientes, a los cuáles invitan a visitar sus explotaciones, a participar de las actividades y a conocer su proyecto social.

En tercer lugar, si observamos los aportes ambientales de estas experiencias observamos que el $70 \%$ de ellas recuperan terrenos o fincas abandonadas a partir de la actividad que desarrollan, mientras que el resto no lo hace por el tipo de actividad que desarrollan, relacionada con el sector de la elaboración agroalimentaria. Por último, y haciendo referencia a los criterios ecológicos presentes en estas entidades, el $70 \%$ de los proyectos analizados realizan una producción ecológica certificada, con sello del Consell Català de la Producció Agrària Ecològica (CCPAE), el organismo regulador en Cataluña. Cabe destacar que, una de las entidades desarrolla su producción aplicando criterios ecológicos pero sin certificación, por discrepancias en el sistema de certificación de la producción ecológica; otra no puede producir completamente en ecológico, aunque es su voluntad en un futuro, por las características del terreno donde se sitúa su actividad; y una tercera solo aplica criterios ecológicos a una de sus líneas de producción. Tan solo una de las 10 entidades no 
aplica ningún criterio de producción ecológica, sin embargo introduce criterios de comercio justo en uno de sus productos.

A continuación mostramos una tabla resumen de las principales características de los modelos de negocio de las entidades seleccionadas (Tabla 3 ).

\begin{tabular}{|c|c|c|c|c|c|}
\hline Entidades & $\begin{array}{l}\text { Estrategia } \\
\text { competitiva }\end{array}$ & $\begin{array}{l}\text { Propuesta de } \\
\text { valor }\end{array}$ & $\begin{array}{l}\text { Relación con el } \\
\text { cliente }\end{array}$ & $\begin{array}{c}\text { Recuperación } \\
\text { del espacio } \\
\text { agrario }\end{array}$ & $\begin{array}{l}\text { Criterios } \\
\text { ecológicos }\end{array}$ \\
\hline L'Ortiga & coste & calidad & $\begin{array}{c}\text { personalizada y } \\
\text { directa }\end{array}$ & $\checkmark$ & $\begin{array}{c}\text { Producción } \\
\text { ecológica } \\
\text { certificada }\end{array}$ \\
\hline $\begin{array}{l}\text { Masia Can Calopa } \\
\text { de Dalt }\end{array}$ & diferenciación & calidad & $\begin{array}{c}\text { personalizada y } \\
\text { directa }\end{array}$ & $\checkmark$ & $\begin{array}{l}\text { Producción } \\
\text { integrada }\end{array}$ \\
\hline $\begin{array}{l}\text { Els Horts de Can } \\
\text { Salas }\end{array}$ & coste & calidad & $\begin{array}{c}\text { personalizada y } \\
\text { directa }\end{array}$ & $\checkmark$ & $\begin{array}{l}\text { Producción } \\
\text { ecológica } \\
\text { certificada }\end{array}$ \\
\hline $\begin{array}{l}\text { Fundació Casa } \\
\text { Dalmases }\end{array}$ & diferenciación & calidad & $\begin{array}{c}\text { personalizada y } \\
\text { directa }\end{array}$ & $x$ & No \\
\hline Bolet Ben Fet & diferenciación & calidad & $\begin{array}{c}\text { personalizada y } \\
\text { directa }\end{array}$ & $x$ & $\begin{array}{c}\text { Producción } \\
\text { ecológica } \\
\text { certificada }\end{array}$ \\
\hline Sambucus & coste & calidad & $\begin{array}{c}\text { personalizada y } \\
\text { directa }\end{array}$ & $\checkmark$ & $\begin{array}{c}\text { Producción } \\
\text { ecológica } \\
\text { certificada }\end{array}$ \\
\hline la Klosca & diferenciación & calidad & $\begin{array}{c}\text { personalizada y } \\
\text { directa }\end{array}$ & $\checkmark$ & $\begin{array}{c}\text { Producción } \\
\text { ecológica } \\
\text { certificada }\end{array}$ \\
\hline $\begin{array}{l}\text { Delícies del } \\
\text { Berguedà }\end{array}$ & coste & calidad & $\begin{array}{c}\text { personalizada y } \\
\text { directa }\end{array}$ & $x$ & $\begin{array}{c}\text { Linias de } \\
\text { producción } \\
\text { diferenciadas }\end{array}$ \\
\hline Riu Verd & coste & calidad & $\begin{array}{c}\text { personalizada y } \\
\text { directa }\end{array}$ & $\checkmark$ & $\begin{array}{c}\text { Producción } \\
\text { ecológica sin } \\
\text { certificar }\end{array}$ \\
\hline $\begin{array}{l}\text { Aprodisca } \\
\text { Ambientals i } \\
\text { Ecològics }\end{array}$ & diferenciación & calidad & $\begin{array}{c}\text { personalizada y } \\
\text { directa }\end{array}$ & $\checkmark$ & $\begin{array}{l}\text { Producción } \\
\text { ecológica } \\
\text { certificada }\end{array}$ \\
\hline
\end{tabular}

Tabla 3 - Principales características del modelo de negocio de las entidades seleccionadas (Fuente: Elaboración propia)

\section{Discusión}

La tendencia al crecimiento de nuevas experiencias de AS en diferentes territorios hace que este fenómeno sea cada vez más importante en el contexto europeo. Sin embargo, la evolución y la dimensión de la AS no aparece como un fenómeno homogéneo en el 
conjunto del continente, sino que subyacen importantes diferencias territoriales, con estadios de desarrollo distintos. Según Di lacovo (2010), se pueden distinguir diversas etapas en la evolución de la AS, desde la novedad del fenómeno, en las que las iniciativas surgen de manera individual con carencia de identidad colectiva y de discurso sobre el fenómeno, hasta la fase de consolidación, cuando los agentes públicos, privados y la sociedad civil reconocen la práctica de la AS y, en consecuencia, existe un discurso bien estructurado, unas directrices claras para el desarrollo de la actividad y una identidad colectiva bien formada que a menudo se deriva en la organización de redes o plataformas que agrupan las diferentes iniciativas. Di lacovo et al. (2014) destaca que la participación de las instituciones a menudo llega en un estadio avanzado de desarrollo, cuando la actividad ya ha alcanzado una importante presencia mediante la participación de la sociedad civil, de las entidades del Tercer Sector Social y, en algunos casos, de obras de beneficencia vinculadas a instituciones eclesiásticas.

La crisis social y económica de 2008 ha motivado la ampliación de los diferentes colectivos beneficiarios de la AS, pasando de centrarse en las personas con discapacidad como principales usuarios a una serie de colectivos (especialmente las personas en situación de pobreza y en paro de larga duración) que han visto afectadas sus necesidades más básicas a raíz de la crisis, un hecho que les ha situado en el umbral de la exclusión social. Estos colectivos que inicialmente eran considerados como potenciales, actualmente podemos observar cómo han ido tomando una mayor presencia. Esta diversidad de colectivos otorga una gran versatilidad al sector que al mismo tiempo exige una mayor especialización en los equipos profesionales debido a las diferentes necesidades específicas, en materia de ocupación, de integración social, educación y tratamiento terapéutico, que cada colectivo requiere.

Los proyectos de AS en Cataluña muestran una diversidad de actividades, que se centran principalmente en la horticultura, especialmente representada actualmente con la incorporación de los huertos sociales. También se observa como la transformación agroalimentaria y los trabajos forestales, junto con la silvicultura, tienen una importancia relevante, quedando menos representados otros sectores como son la ganadería y la artesanía. Gran parte de estas experiencias elaboran sus productos mediante criterios 
ecológicos, contribuyendo a dibujar un modelo de producción más sostenible y un desarrollo económico más justo, ético y solidario. La AS emerge como respuesta, desde la innovación social, a ciertas necesidades comunes de varios colectivos, poniendo en valor la función social de la agricultura, e impulsando sistemas de producción alternativos al modelo agroindustrial. La AS pretende construir alternativas económicas con valor social añadido, una práctica asociada a la producción local y ecológica y el surgimiento de formas de consumo responsable entre sectores cada vez más amplios de la sociedad.

Al analizar la dimensión ambiental de la AS, observamos que es un fenómeno eminentemente periurbano, que ofrece la oportunidad de recuperar y dar valor a los espacios agrícolas que han sido abandonados o descuidados, al recibir una presión por su cercanía a la ciudad. El desarrollo de la AS favorece el esponjamiento de los sistemas territoriales urbanos, y contribuye al aumento de la biodiversidad en los entornos donde se implementa, reduciendo la erosión de los espacios agroforestales y una minimización del riesgo de incendio. Los proyectos localizados en áreas más rurales, a parte de estos beneficios ambientales, nos permiten comprobar la aportación de la AS al Desarrollo Local Sostenible, relacionando los recursos locales, actividades y actores de un mismo territorio para generar practicas innovadoras y productos con un alto valor añadido que revaloricen el territorio y creen nuevas oportunidades. Al basarse la AS en una producción agraria local y una comercialización "de proximidad" puede convertirse en un elemento clave alrededor del cual se organicen ciertas estrategias locales.

\section{Conclusiones}

La AS en Cataluña se encuentra en una etapa inicial de desarrollo, aunque existen proyectos desde la década de 1970 y 1980, como L'Olivera y La Fageda, superando ya el estadio de novedad, y siendo un sector con un importante crecimiento. Progresivamente van apareciendo nuevas experiencias y redes que ayudan a consolidar esta práctica, a la vez que aumenta su visibilidad social y su reconocimiento por parte de la administración, aunque la ayuda de esta haya disminuido recientemente. La AS contribuye a empoderar y generar 
oportunidades de ocupación para personas en riesgo de exclusión social, mejorando la calidad de vida, la autonomía y la inclusión en la sociedad de los grupos con mayor vulnerabilidad. A nivel individual, el trabajo en las entidades de AS genera importantes mejoras en la salud física y mental de las personas, así como un mayor bienestar emocional, aumentando la autoestima y la confianza en sí mismos.

La AS aparece como una clara práctica de innovación social, relacionada con la mutualidad, la acción colectiva, la cohesión y la interacción activa de las personas a nivel particular, y familiar. Se utilizan los recursos locales de forma comunitaria con una escasa interacción del estado. Estas características definen el sistema de bienestar mediterráneo donde el papel de la sociedad civil ocupa un lugar muy importante (Nadini, 2003). A parte de esto, la AS subyace como una estrategia de resiliencia para generar "nuevas" economías locales, más centradas en las especificidades, las potencialidades y las oportunidades de cada territorio, que en las tendencias y la conectividad con la economía global (Estela, 2015).

La AS, como actividad de la economía social, solidaria y cooperativa, deja de lado los planteamientos únicamente económicos y se fija en cómo pueden utilizarse los recursos locales para generar un desarrollo económico resiliente, que contribuya a alcanzar un mayor bienestar individual y colectivo y promueva la sostenibilidad económica, pero también la ambiental, social y territorial (Walker y Salt, 2006). Entonces la AS se presenta de forma distinta en cada territorio, heterogeneidad resultado de las estrategias de resiliencia para dar respuesta a las necesidades de la sociedad y adaptarse a los cambios socioeconómicos.

\section{Agradecimientos}

Esta investigación ha sido realizada por el Equip de Recerca en Agricultura Social ${ }^{1}$ del Departament de Geografia de la Universitat Autònoma de Barcelona (ERAS-UAB) integrado por Antoni F. Tulla (IP), Anna Badia, Ana Vera, Àngel Cebollada, Carles Guirado, Natàlia Valldeperas (Departament de Geografia, UAB), Josep Espluga (Departament de Sociologia

\footnotetext{
${ }^{1}$ Página web del grupo de investigación: https://agriculturasocialcat.wordpress.com/
} 
UAB), Imma Pallarès, Camille Evard y Laia Sendra (técnicas autónomas, antes CEDRICAT), y se ha financiado con los proyectos CSO2012-31979, 2011ACUP023 y 2014ACUP029 (Convocatorias de RecerCaixa 2011 y RecerCaixa 2014).

\section{Referencias}

ARMESTO, Xosé Antón (2005): Notas teóricas en torno al concepto de postproductivismo agrario. Investigaciones Geográficas, nㅇ36, p. 137-156.

ARQUÉ, Maite, GARCÍA, Àngela and MATEU, Xavier (1982): La penetració del capitalisme a les comarques de I'Alt Pirineu. Documents d'Anàlisi Geogràfica, no 1, p. 9-67.

BARRACHINA, Maria, GUIRADO, Carles and TULLA, Antoni F. (2009): Viejos problemas, nuevas respuestas: las áreas de montaña frente a las debilidades del sistema neoliberal. El caso del Pirineo Catalán. En PILLET, Félix; CAÑIZARES, Maria C and RUIZ, Ángel R. (coord.). Geografía, territorio y paisaje: el estado de la cuestión: actas del XXI congreso de geógrafos españoles, Ciudad Real 27-29 de octubre de 2009. Ciudad Real: Asociación de Geógrafos Españoles, p. 85-97.

BELL, David. (2006): Variation on the rural idyll. En CLOKE, Paul, MARSDEN, Terry, MOONEY, Patrick H. (eds) The Sage Handbook of Rural Studies. London: Sage Publications, p. 149-160.

BINIMELIS, Jaume and ORDINAS, Antoni. (2012): Agricultura y postproductivismo en las Islas Baleares. La Payesía isleña en los albores del siglo XXI, Scripta Nova, no XVI, p. 393.

BOWLER, Ian R, BRYANT, Christopher and COCKLIN, Chris. (2002): The sustainability of rural systems: Geographical interpretations. Dordrecht: Kluwer academics Pubs.

CARBONE, Anna, GAITO, Marco and SENNI, Saverio. (2007): Consumers' Buying Groups in the Short Food Chains: Alternatives for Trust. En 1st International European Forum on Innovation and System Dynamics in Food Networks. Innsbruck-Igls, Austria: February, p. 15-17.

CLOKE, Paul. (2006): Conceptualizing rurality. En Cloke, Paul. Marsden, Terry, Mooney, Patrick H. (eds), The Sage Handbook of Rural Studies. London: Sage Publications, p. 18-28.

DI IACOVO, Francesco. (2010): L'agricoltura sociale: pratiche e paradigmi nello scenario comunitario, En: XLVII Convegno di Studi de la Società Italiana di Economia Agraria. Università degli Studi del Molise. Campobasso.

DI IACOVO, Francesco, MORUZZO, Roberta, ROSSIGNOLI, Cristiano and SCARPELLINI, Paola. (2014): Transition management and social Innovation in rural areas: Lessons from social farming, The Journal of Agricultural Education and Extension, no 20, p. 327-347.

DI IACOVO, Francesco and O'CONNOR, Deirdre (2009): Supporting Policies for Social Farming in Europe. Progressing Multifunctionality in Responsive Rural Areas. So Far. Social Services in Multifunctional Farms Farms ("Social Farming"). Florencia: ARISA.

DUCH, Gustavo. (2010): Lo que hay que tragar: minienciclopedia de política y alimentación. Madrid: Los Libros de Lince.

ESTELA, Oriol. (2015): Politiche pubbliche per economie locali resilienti. Territorio della Ricerca su Insediamenti e Ambiente. Rivista internazionale di cultura urbanistica, $\mathrm{n} \cong 15, \mathrm{p} .81-94$.

Gazzetta Ufficiale de la Repubblica Italiana, Legge 18 agosto 2015, n. 141: Disposizioni in materia di agricultura sociale. http://www.gazzettaufficiale.it/eli/id/2015/09/8/15G00155/sg.

GUIRADO, Carles. (2011): Tornant a la muntanya. Migració, ruralitat i canvi social al Pirineu català. El cas del Pallars Sobirà. Tesi doctoral. Bellaterra (Cerdanyola del Vallès): Universitat Autònoma de Barcelona. 
GUIRADO, Carles, BADIA, Anna, TULLA, Antoni F, VERA, Ana and VALLDEPERAS, Natàlia. (2014): La agricultura social en Catalunya: innovación social y dinamización agroecológica para la ocupación de personas en riesgo de exclusión. Ager, Revista de estudios sobre despoblación y desarrollo rural, no 17, p. 65-97.

GUIRADO, Carles and TULLA, Antoni F. (2010): Entre l'abandonament i l'ús intensiu del territori? Sistema d'assentaments i gestió del territori en espais de muntanya. El cas de l'Alt Pirineu Català, Documents d'Anàlisi Geogràfica, 56 (3), 607-623.

HALVELL, B. (2002): Home Grown. The case for local food in a Global Market. En Prough, T. (ed) Worldwatch Paper 163, Danvers (Ma) USA.

LECK, Chris, EVANS, Nick and UPTON, Dominic. (2014): Agriculture - who cares? An investigation of 'Care Farming' in the UK. Journal of Rural Studies, nㅇ 34, p. 313-325.

LOCKWOOD, Jeffrey A. (1999): Agriculture and biodiversity: finding our place in this world. Agriculture and Human Values, no 16, p. 365-379.

LÓPEZ, José A, and FERNÁNDEZ, Ramón (2003): Con la comida no se juega: Alternativas autogestionarias a la globalización capitalista desde la agroecología y el consumo. Madrid: Traficantes de Sueños.

MARTINEZ-TORRES, María E and ROSSET, Peter M. (2010): La Vía Campesina: the birth and evolution of a transnational social movement. The Journal of Peasant Studies, no 37 (1), p. 149-175.

MONLLOR, Neus (2013): La nova pagesia: vers un nou model agrosocial. Quaderns Agraris, no 35, p. 7-24.

NALDINI, Manuela. (2003): The family in the Mediterranean welfare states. London: Routledge.

NOGUÉ, Joan. (1988): El fenómeno neorrural. Agricultura y Sociedad, no 47, p. 145-175.

PALLARÈS-BLANCH, Marta, TULLA, Antoni F. and VERA, Ana. (2015): Environmental capital and women's entrepreneurship: A sustainable local development approach. Carpathian Journal of Earth and Environmental Sciences, no 10 (3), p. 133-146.

PORTER, Michael E. (1985): The Competitive Advantage: Creating and Sustaining Superior Performance. New York: Free Press.

SCHNEIDER, Stephen. (2008): Good, Clean, Fair: The Rhetoric of the Slow Food Movement. College English, no70 (4), p. 384-402 (Special Focus: Food).

TULLA, Antoni F, PALLARÈS-BARBERÀ, Montserrat and VERA, Ana. (2009): Naturbanization and local development in the mountain areas of the Catalan Pyrenees. En Prados, M. J. (ed) Naturbanization: New identities and processes for rural-natural areas. Londres: Taylor \& Francis Group, p. 75-92.

TULLA, Antoni .F, VERA, Anna, BADIA, Anna, GUIRADO, Carles and VALLDEPERAS, Natàlia. (2014): Rural and Regional development policies in Europe: Social Farming in the Common Strategic Framework (Horizon 2020). Journal of Urban and Rural Analysis, no VI (1), p. 35-52.

TULLA, Antoni F, GUIRADO, Carles, BADIA, Anna, VERA, Ana, VALLDEPERAS, Natàlia and EVARD, Camille. (2015): L' Agricultura Social a Catalunya. Uma doble alternativa: Desenvolupament local $i$ ocupació de col/lectius en risc d'exclusió social. Quaderns Agraris, no 38, p. 23-49.

VAN DER PLOEG, Jan D. (2006). Agriculture production in crisis. En Cloke, P., Marsden, T., Mooney, P. (eds) Handbook of Rural Studies. Londres: Sage Publications, p. 258-277.

VERA, A, BADIA, Anna, TULLA and Antoni F. (2011): Desarrollo local en el Pirineo Catalán: impulso económico y uso sostenible del territorio. Finisterra: Revista Portuguesa de Geografia, no XLVI, 92, p. 9-27.

WALKER, Brian and Salt, David. (2006): Resilience Thinking: Sustaining Ecosystems and People in a Changing World. New York: Island Press.

WOODS, Michael (2005): Rural Geography: processes, responses and experiences in rural restructuring. Londres: Sage Publications.

WOODS, Michael (2011): Rural. Abingdon: Routledge. 